\title{
EL PROGRAMA ICONOGRÁFICO LA BASÍLICA DE NUESTRA SEÑORA DE LAS ANGUSTIAS DE GRANADA: LOS CICLOS PICTÓRICOS DE LOS ANTECAMARINES (1739-1742) ${ }^{1}$.
}

\author{
NURIA MARTÍNEZ JIMÉNEZ. \\ Universidad de Granada.
}

Resumen

En la primera mitad del siglo XVIII el gusto por la ornamentación mural se extendió por los edificios más relevantes de Granada alentado por las órdenes religiosas y por la nobleza. En este ambiente artístico, Juan de Lizana decidió impulsar la pintura de los antecamarines de la Basílica de Nuestra Señora de las Angustias de Granada, para ensalzar el papel ejemplarizante de María, acorde con los parámetros contrarreformistas. Este artículo pretende mostrar los resultados de las últimas investigaciones realizadas sobre la extraordinaria decoración pictórica de los antecamarines, que a su vez se constituye como un recurso histórico de primer orden para comprender este fascinante periodo histórico.

Palabras clave

Pintura mural. Iconografía. Joseph Hidalgo. Juan de Medina. Basílica de Nuestra Señora de las Angustias. Granada. Siglo XVIII.

\section{THE ICONOGRAPHIC PROGRAMME FROM THE BASÍLICA OF ANGUSTIAS: THE PICTORIAL DECORATION OF THE SAFE- KEEPING ROOMS (1739-1742)}

\begin{abstract}
In the first decades of the 18th Century wall paintings decoration was used in the most important buildings of Granada through the support of religious ordenes and nobility. In this atmostphere, Juan de Lizana decided to paint the safe-keeping room of the Basilica of Angustias.

In conclusion, this article shows the latest researches about the decoration from the Basilica of Angustias to explain the symbolism and the importance of this historical source.
\end{abstract}

\section{Keywords}

Mural painting. Iconography.Joseph Hidalgo. Juan de Medina. Basilica of Angustias. Granada. 18th century.

\footnotetext{
${ }^{1}$ Este artículo se inscribe en el Proyecto I+D: «La copia pictórica en la monarquía hispánica (siglos XVIXVII)». Programa estatal de fomento de la investigación científica y técnica de excelencia. Referencia: HAR2014-52061-P. (Fecha de concesión 2015)
} 


\section{Introducción}

"Nobie Marmoreo, culto aquí erigido./ Con la pluma, y pincel se ve animado/Los antecamarines dan unido /Desta Reina lo triste, y Angustiado;/Profetico el primero a establecido/Los Misterios que el otro ha demostrado/Siendo los dos, y el trono a su Caricia/ Un tributo ofrecido de Justicia 2 ".

El camarín de la Basílica de la Virgen de las Angustias de Granada fue levantado a finales del siglo XVII. El diseño de la planta fue realizado por Juan de Mena en $1703^{3} \mathrm{y}$, durante el proceso constructivo, sufrió diversas modificaciones con el fin de mantener la homogeneidad de la iglesia. En ese sentido una de las innovaciones más relevantes la introdujo el arquitecto cordobés Francisco Hurtado Izquierdo en $1712^{4}$, al recurrir a las maderas policromadas para imitar los coloridos mármoles del retablo. A través de esta solución consiguió aligerar el peso de la estructura y reducir los costes, por lo que esta idea la mantuvieron sus sucesores Baltasar de la Pasión y Bernardo de Valdivia.

Las dificultades económicas de la obra explican las constantes fluctuaciones en el proceso constructivo, así como la extraordinaria relevancia del impulso ofrecido por Don Juan de Lizana a partir de $1730^{5}$. Gracias a su patrocinio se doraron los capiteles, se colocó la solería del templo con mármoles de Macael y Sierra Nevada y, además, Marcos Fernández Raya concluyó el extraordinario retablo realizando el magnífico trono que, a modo de peana, contribuyó a ensalzar la imagen de la Virgen.

De esta forma, concluidas las obras arquitectónicas y escultórica de las naves de la iglesia y del camarín, se dio pasó al ornato pictórico.

\section{Precedentes en el estudio del ornato pictórico de los antecamarines.}

Desde la construcción del camarín de la Basílica de Nuestra Señora de las Angustias, éste ha suscitado gran interés desde el punto de vista historiográfico.

Las primeras noticias las ofrece el pintor y académico granadino José Sánchez Sarabia, cuando indica "a uno y otro lado de este arquitectónico prodigio brilla el decoro de los dos ante camarines de bella idea, descuello, y amplitud, ilustrado todo el ámbito de sus lienzos de pared, y bóvedas con primorosas pinturas de pincel muy sabio, alusivas a las Angustias y Dolores de nuestra gran Madre"

Décadas después, José Giménez Serrano, cambia su perspectiva obviando la decoración pictórica de los ante camarines, y centrándose en la obra arquitectónica al describirlas con estas palabras: "El camarín y el retablo principal dirigidos y trazados por Don Pedro Duque de Cornejo, son como suyos, y causa compasión ver tan ricos mármoles amontonados y tallados con tan extraviado gusto ${ }^{8}$.

Más allá de estas descripciones la línea que sin duda ha definido los estudios sobre el camarín y los ante camarines parten de los textos del historiador Manuel Gómez Moreno expresados principalmente en la Guía de Granada. Concretamente sobre las pinturas de los muros de los ante camarines, indica lo siguiente "E camarín hecho al par de la iglesia, fue posteriormente decorado con inusitada suntuosidad y se terminó en 1742 a costa de los devotos". "Las habitaciones laterales fueron al mismo tiempo decoradas con grandes

\footnotetext{
${ }^{2}$ Cartela a los pies de San Fernando. Antecamarín del lado de la Epístola. Basílica de Nuestra Señora de las Angustias.1742. Granada.

${ }^{3}$ López Guadalupe/ López Guadalupe, 1996: 150-151.

${ }^{4}$ Ibídem.: 151.

5 Ibid::157.

${ }^{6}$ Se refiere al camarín.

7 Sánchez Sarabia, 1777: 28.

${ }^{8}$ Giménez Serrano, 1846: 333.

${ }^{9}$ Goméz Moreno, 1982: 237.
} 


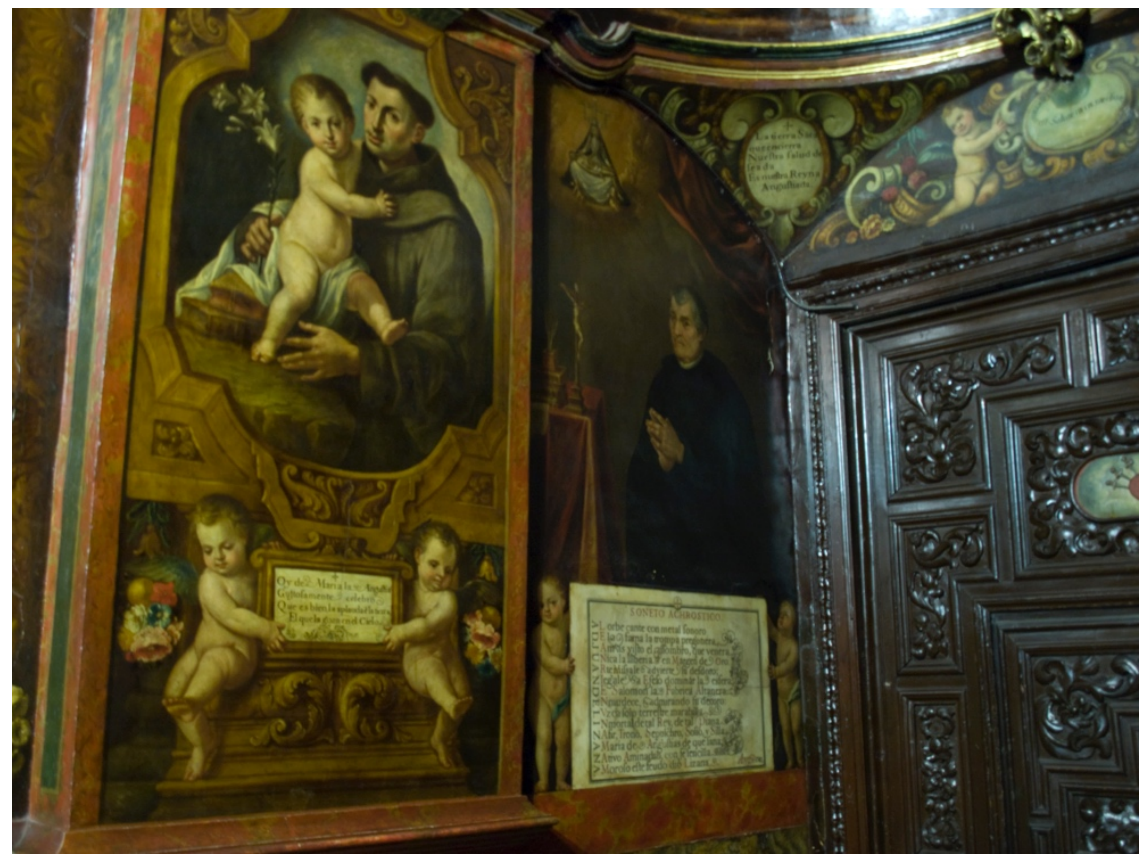

Fig. 1. José Hidalgo y Juan de Medina. Entrada al antecamarin. Retrato de Juan de Lizana. Basílica de Nuestra Señora de las Angustias, Granada.

pinturas al óleo sobre la pared, que representan los dolores de Nuestra Señora y otros pasajes de su vida, hechas con facilidad y bastante apreciables para aquellos tiempos ${ }^{10,}$.

Años después, en su Guía ${ }^{11}$, Antonio Gallego Burín indica fecha y la autoría de las pinturas "las salas laterales, decoradas con pinturas al óleo de los Dolores de la Virgen y pasajes de su vida, hechas, de 1739 a 1741, por José Hidalgo y terminadas, en 1742, por Juan Medina”.

Poco después María Jesús Chavarino García realizó una tesis que actualmente es poco conocida y cuyo valor radica en la transcripción documental y de los textos que aparecen en el camarín ${ }^{12}$. Tras estas citas, la mayoría de textos que abordan la Basílica de Nuestra Señora de las Angustias se centrarán en analizar los aspectos arquitectónicos y escultóricos de la misma, destacando primordialmente los estudios sobre la imagen de Nuestra Señora de las Angustias $^{13}$. En efecto, habrá que esperar una década hasta la publicación de la obra monográfica de Juan José y Miguel Luis López Guadalupe para leer el primer acercamiento a las pinturas. En este libro los investigadores abordan en profundidad los aspectos históricoartísticos que más relevantes de la Basílica y entre ellos destacan el camarín y los antecamarines. Desde el punto de vista del análisis pictórico introducen los temas narrativos que discurren por las paredes de los ante camarines, transcribiendo los textos más significativos e incitan a profundizar en el estudio de las mismas ${ }^{14}$. En este lugar es donde se encuentra este artículo, fruto del trabajo de investigación tutelada titulado "Juan de Medina un muralista en la Granada de la Edad Moderna: estado de la cuestión" dirigido por Dr. D. Antonio Juan Calvo Castellón y del cual dimana este artículo.

La decoración pictórica de los antecamarines.

\footnotetext{
10 Ibidem.: 238.

11 Gallego Burín, 1982:193.

12 Chavarino García, 1986.

${ }^{13}$ Entre las obras es imprescindible citar a Antequera, 1973. Gutierrez Galdo, 1983. Isla Mingorance, 1989.

${ }^{14}$ López Guadalupe/López Guadalupe, 1996:162.
} 
El exquisito tratamiento de los mármoles y maderas policromadas del camarín contribuyó a crear un espacio que parecía sobrepasar los límites reales, ensalzados por el reflejo dorado de la luz que penetraba por los vanos de la imponente cúpula. Con todo, se otorgó a los antecamarines un papel protagonista en el programa iconográfico de este espacio a través de su ornato pictórico.

La tradición de la pintura mural en Granada hunde sus raíces en los albores del siglo XVI. Pero no fue hasta la primera mitad del siglo XVIII cuando alcanzó su cénit. De hecho, la estela iniciada en 1712 por la sublime paleta de Antonio Palomino, ayudado por José Risueño en la cúpula del Sancta Sanctórum de la Cartuja, motivó la difusión de esta técnica hasta llenar de color las fachadas y los interiores de los edificios civiles y religiosos más relevantes de la capital.

En este contexto se explica el interés prestado por Juan de Lizana en la decoración pictórica de los antecamarines de la iglesia, una de las construcciones más emblemáticas de la ciudad gracias al fervor popular que suscitaba la Virgen de las Angustias.

Originariamente, estas estancias servían para distribuir los donativos de los fieles. Sin embargo, paulatinamente fueron incrementando su importancia hasta convertirse en una parte indisoluble del mirabile composito del camarín, en el que arquitectura, escultura y pintura conforman una obra de arte total.

En ese sentido tras la participación de los más excelsos arquitectos y escultores que estaban trabajando en la ciudad, el colofón de la obra lo constituiría la decoración pictórica de estas estancias. Esto explica que la culminación de estos trabajos en el camarín, que habían sido iniciados el 11 de mayo de $1739^{15}$ y culminados "el 23 de septiembre de 1742 con un gasto total de 153111 reales con 15 maravedíes $^{16 ", ~ d i e r a ~ p a s o ~ a l ~ e l e n c o ~ d e ~ f i e s t a s ~ y ~}$ celebraciones encargadas por Lizana para celebrar la culminación de las obras de la iglesia ${ }^{17}$.

Los artífices de este proyecto fueron Joseph Hidalgo y Juan de Medina. La trayectoria personal y artística de Joseph Hidalgo, es prácticamente desconocida, pero su notoriedad se evidencia en la destreza técnica empleada en las composiciones murales del Ciclo de la Infancia de Jesú ${ }^{18}$; un excelente trabajo por el que percibió un salario 20 reales diarios ${ }^{19}$ y en el que estuvo acompañado por sus oficiales Francisco Marchena y, posteriormente, Antonio Rodríguez ${ }^{20}$.

La homogeneidad lograda en el programa iconográfico y la convivencia temporal de los artistas, plantea la premeditación de un trabajo compartido. Sin embargo, el hecho de que Medina cobrara 18 reales diarios (2 menos que su antecesor) supuso una considerable reducción de los gastos sin mermar la excelencia y la calidad de las pinturas. En las primeras décadas del siglo XVIII el pintor Juan de Medina, se había ensalzado como uno de los muralistas más influyentes y prolíficos de Granada ${ }^{21}$. Notoriedad que motivaría su participación en esta obra iniciada, junto con su oficial ${ }^{22}$, el 13 de mayo de 1741.

El primer trabajo conocido de Juan de Medina es la participación en la decoración pictórica de la Iglesia del Monasterio de San Jerónimo, realizada al fresco entre los años 17231735, junto con otros artistas como Martín de Pineda. En esta obra, Medina tuvo la

${ }^{15}$ Libro de gastos de la obra del camarín. Desde 1711 a 1742. Archivo de la Hermandad de las Angustias (AHA) Granada. s.n.

${ }^{16}$ De los cuales 16146 reales hacen referencia sólo a la pintura.

${ }^{17}$ Libro de gastos de la obra del camarin. Desde 1711 a 1742. AHA. Granada.. s.n..

${ }^{18}$ En la documentación no se detallan las composiciones realizadas por cada maestro. Sin embargo, consideramos que este ciclo se adecua a la cronología de sus trabajos. Además, por analogías técnicas y artísticas con otras obras consideramos que las composiciones del Ciclo de la Pasión fueron realizadas por de Juan de Medina.

${ }^{19}$ En total el salario percibido por el pintor ascendió a 9520.

${ }^{20}$ Que sustituyó al anterior en noviembre de 1740.

$21 \mathrm{Al}$ año siguiente decoró el nicho del coro del convento de las Agustinas (1743). En última instancia es importante incluir su labor como muralista en el Convento de la Concepción y la realización de obras sobre caballete como el Árbol Hospitalario del Hospital de San Juan de Dios.

${ }^{22} \mathrm{Y}$ el "que muele "y después "oficial” 5 reales por día. Juan Medina también realizó tareas como "maestro dorador". Libro de gastos de la obra del camarín. Desde 1711 a 1742. AHA. Granada.. s.n.. 
oportunidad de demostrar su pericia ${ }^{23}$ y adquirir la experiencia suficiente para ensalzarse como el muralista idóneo para continuar en el programa iniciado por Hidalgo y cumplir con los objetivos del cliente. Como resultado se realizó el ornato de las estancias aprovechando las ventajas ofrecidas por la técnica del óleo sobre muro ${ }^{24}$ para conformar el discurso de cada estancia.

\section{Programa iconográfico.}

Las pinturas ocupan cada milímetro de estas pequeñas estancias rectangulares localizadas en un nivel intermedio entre el piso de la nave y el camarín, por ello las composiciones han de adaptarse al marco que las sustenta con el fin de crear una visión unitaria de todo el conjunto sin desvirtuar el mensaje.

De este modo, el recorrido se inicia en el estrecho pasillo que da acceso al antecamarín del lado de la Epístola con el Ciclo de la infancia y madurez de Jesús, y se concluye en el lado del Evangelio con las escenas que recogen la Pasión, muerte y resurrección de Cristo.

El antecamarín del lado de la Epístola: Infancia y madurez de Jesús (1739-1741).

Como prólogo al ciclo iconográfico en esta primera estancia las representaciones y las cartelas explican las razones por las cuales Juan de Lizana impulsó está decoración pictórica.

La primera pintura de la sala es el retrato orante del patrocinador ataviado con sus hábitos eclesiásticos frente a una mesa donde hay una Biblia, un tintero y un crucifijo. Su mirada se dirige hacia el espectador apoyado, simbólicamente, por la presencia Virgen de las Angustias envuelta en un halo dorado. Para apoyar la pintura en la parte inferior se lee el siguiente soneto acróstico:

"Soneto achrostico

AL orbe cante con metal sonoro

DE la fama la trompa pregonera,

JAmas visto el assombro, que venera

UNica la Iliberia en Margen de Oro.

ARtemissa le advierte tu desdoro;

NIegale a Efeso dominar la esfera;

DE Salomón la Fabrica Altanera

ENmudece, admirando su decoro:

LUzca solo terrestre marabilla

INmortal de Tal Rey, de tal Diana

ZAfir, Trono, Sepulchro, Solio y Silla

A María de Angustias de que sana;

NAtivo Aminadab, con fe sencilla

AMoroso este feudo dio Lizana. Ano de 1742".

Con el fin de reafirmar la importancia de la empresa, en el estrecho pasillo, se representan cuatro santos especialmente significativos, que ofrecen una valiosa información sobre el proceso decorativo.

El primero de ellos es San Antonio cuya onomástica ${ }^{25}$ se puede relacionar con el día que concluyó el ornato pictórico, el 23 de septiembre. Además lo relata así el texto "(H)Oy de María la Angustia/Gustosamente celebro/que es bien, la aplauda e(n)la tierra/El que la goza en el Cielo".

\footnotetext{
${ }^{23}$ En este punto es conveniente mencionar que los trabajos de Medina en San Jerónimo los realizó al fresco, mientras que en los antecamarines continua la técnica de óleo sobre temple iniciada por José Hidalgo.

${ }^{24}$ En la actualidad los colores se han visto oscurecidos por su propia naturaleza y como consecuencia de las restauraciones realizadas entre 1916 y 1917 motivadas por un incendio.

25 En algunas zonas de Granada.
} 


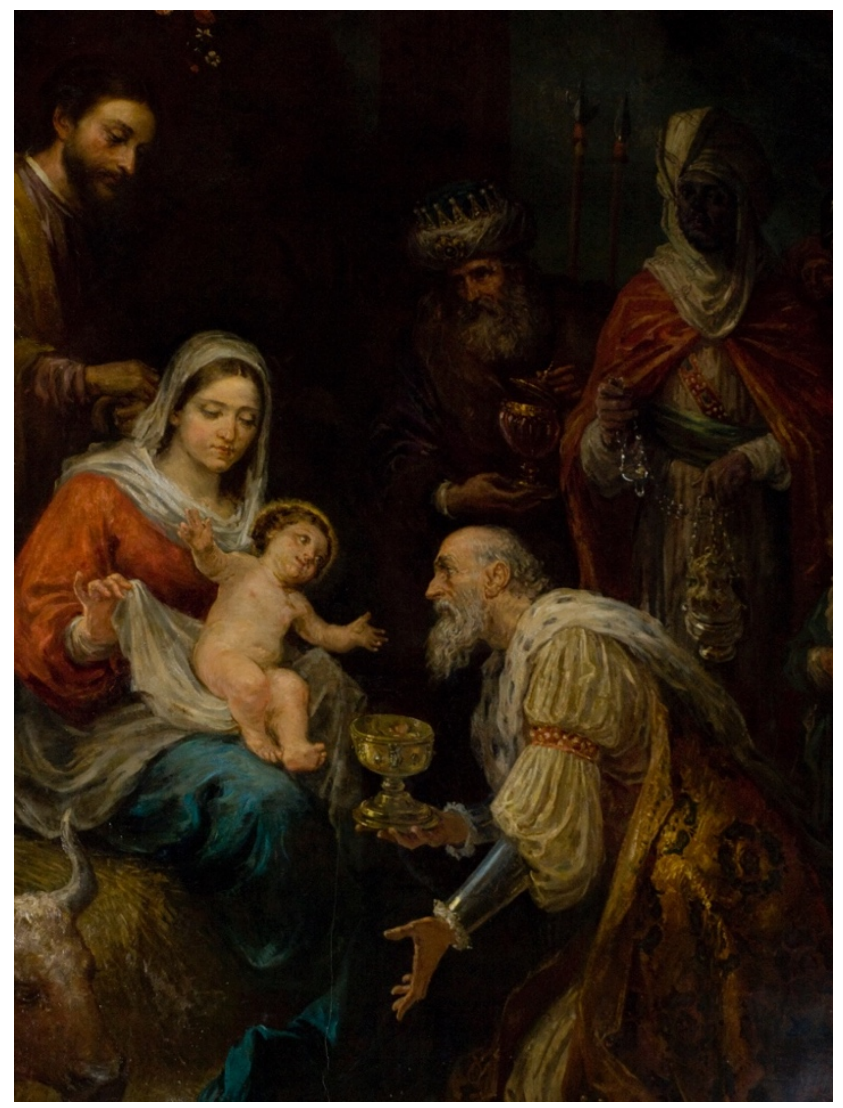

Fig.2. José Hidalgo y Juan de Medina. Epifanía. Basílica de Nuestra Señora de las Angustias, Granada.

En la pared de enfrente se encuentra San Fernando, en una clara alusión al futuro rey Fernando VI que en 1730 declaró el Real Patronato de la iglesia y se proclamó hermano de la Real Hermandad de Nuestra Señora de las Angustias. Más allá de la apariencia real y los atributos que lo acompañan (palma, llaves) lo más destacado de esta escena es el texto de la cartela, donde explica la decoración de los antecamarines y que aparece transcrito al inicio del texto.

El último tema representado es San Juan Bautista acariciando tiernamente al Cordero que se encuentra recostado junto a la cruz y cuya presencia se explica así: "Un noble deboto afecto/con mi nombre distinguido/para Gloria tuya, y mía/me coloca en este sitio".

Una vez expresada la motivación principal de la realización de las pinturas da comienzo el ciclo de la Infancia y la madurez de Jesús, concretado en una serie de episodios que profetizan la tristeza y las angustias de María, y organizado en torno al arco monumental que da acceso al camarín.

El discurso se inicia con la representación de San Miguel venciendo al diablo. Una escena extraída del Apocalipsis de San Juan que está directamente vinculada con la Inmaculada Concepción de María.

Sobre la bóveda del pasillo se halla La Anunciación del Ángel Gabriel a María apoyada por el texto que dice así: "Gabriel anunció//esta Gloria//y previene con con//tento. Esta Reina al Sentimiento". Así mismo, inspirados por el texto de Lucas los artistas presentan al arcángel Gabriel y la Virgen María arrodillados entorno al lirio blanco de la pureza y al jarrón de azucenas sostenido por dos angelitos alados. Todo ello supervisado por la figura de Dios Padre.

Seguidamente se descubre una de las escenas más bellas de todo el programa: la Epifanía. Un episodio frecuentemente representado como muestra del reconocimiento del Mesías por otros pueblos, y por tanto, de la extensión universal de la esperanza redentora. 


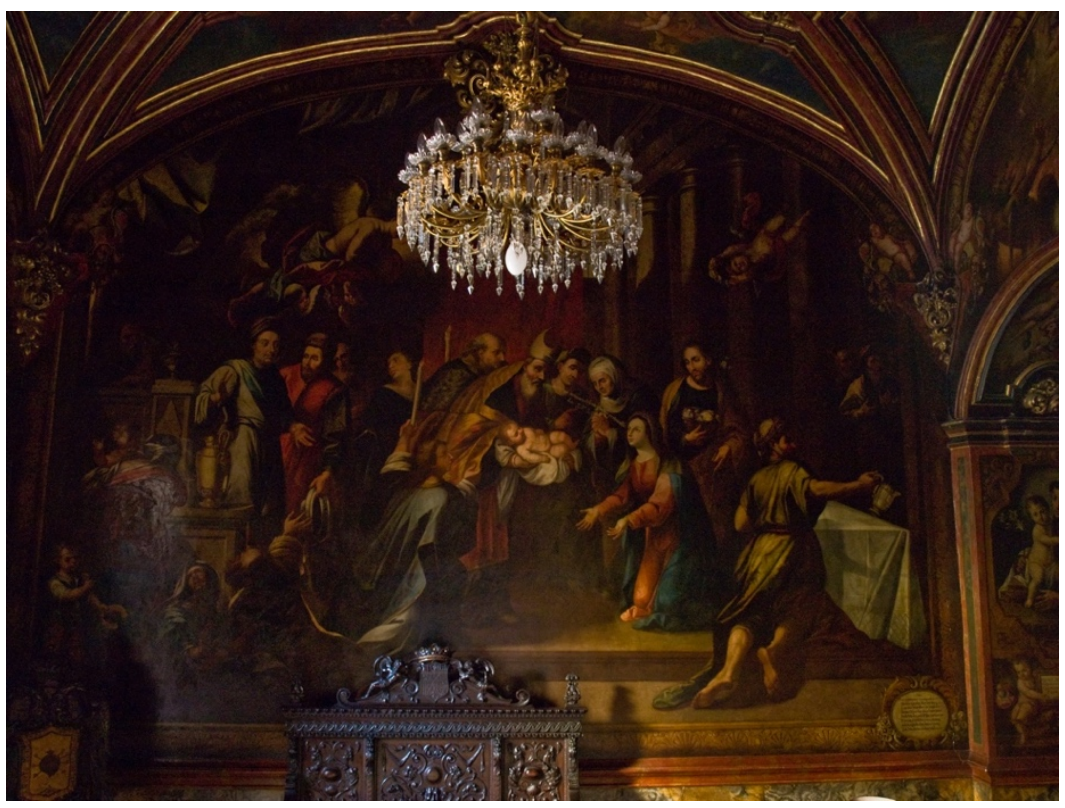

Fig. 3. José Hidalgo y Juan de Medina. La Presentación del Templo. Basílica de Nuestra Señora de las Angustias, Granada.

En el centro de la composición se aprecia la Sagrada Familia y los Reyes de Oriente. Primeramente se presenta Melchor, despojado de su corona real, arrodillado frente al Niño y ofreciéndole la copa con el oro; Baltasar permanece de pie sujetando el incensario junto a un pequeño paje y Gaspar destapa la copa de mirra. Sobre ellos, dos ángeles vuelan graciosamente arrojando flores y mostrando una filacteria donde se lee "Obtulerum Por oR(o)Et Myrrham ${ }^{26 \prime}$. A los pies de la escena la octava relata:

"Ya no luce la estrella en el Oriente//Que el rostro de jesus la eclipsaría/Brillando como el Sol resplandeciente/Tras el horror de tempestad sombría;//Tanto, que absorta la piadosa gente/Que el remoto confín del Asia envía, Mientras le rinde adoración de hinojos $\mathrm{Ne}(. .$.$) parar los ojos".$

Seguidamente se representan dos ceremonias rituales de la Ley Mosaica que debían cumplirse tras el parto: Presentación al templo y la Circuncisión ${ }^{27}$.

La Presentación del Templo ${ }^{28}$ es la composición de mayores dimensiones de todo el programa. La ceremonia se ubica en un espacio arquitectónico definido por las columnas y enmarcado por un gran telón sostenido por dos ángeles. En el centro María recibe al Niño de manos de Simeón diciendo "Et tuam plius amara doloris gladis pertran filio" ${ }^{29 "}$. Junto a ella aparece José, con los dos pichones, y otros dos personajes entre los que podría estar la Profetisa Ana. Por el tamaño del muro en el que se encuentra, una vez dispuestos los protagonistas esenciales, los artistas disfrutaron de cierta libertad compositiva para desarrollar su técnica incorporando fuertes escorzos e incluyendo escenas naturalistas. En esta ocasión el texto de la octava dice así:

"Dos coraçones una espada fiera/de dos agudos filos se traspasa/Los dos veo, si bien se Considera/forman, Y por los dos el uno pasa/De la Madre, y del Hijo común era

\footnotetext{
26 "Ofrecieron oro y mirra". Math2.

27 Recogidos en el Pentateuco: Levítico 12,1-7 y Éxodo 13,1-2.

${ }^{28}$ Lc, 2,21-24.

29 "Y la espada más amarga de la tristeza pasó a su hijo".
} 


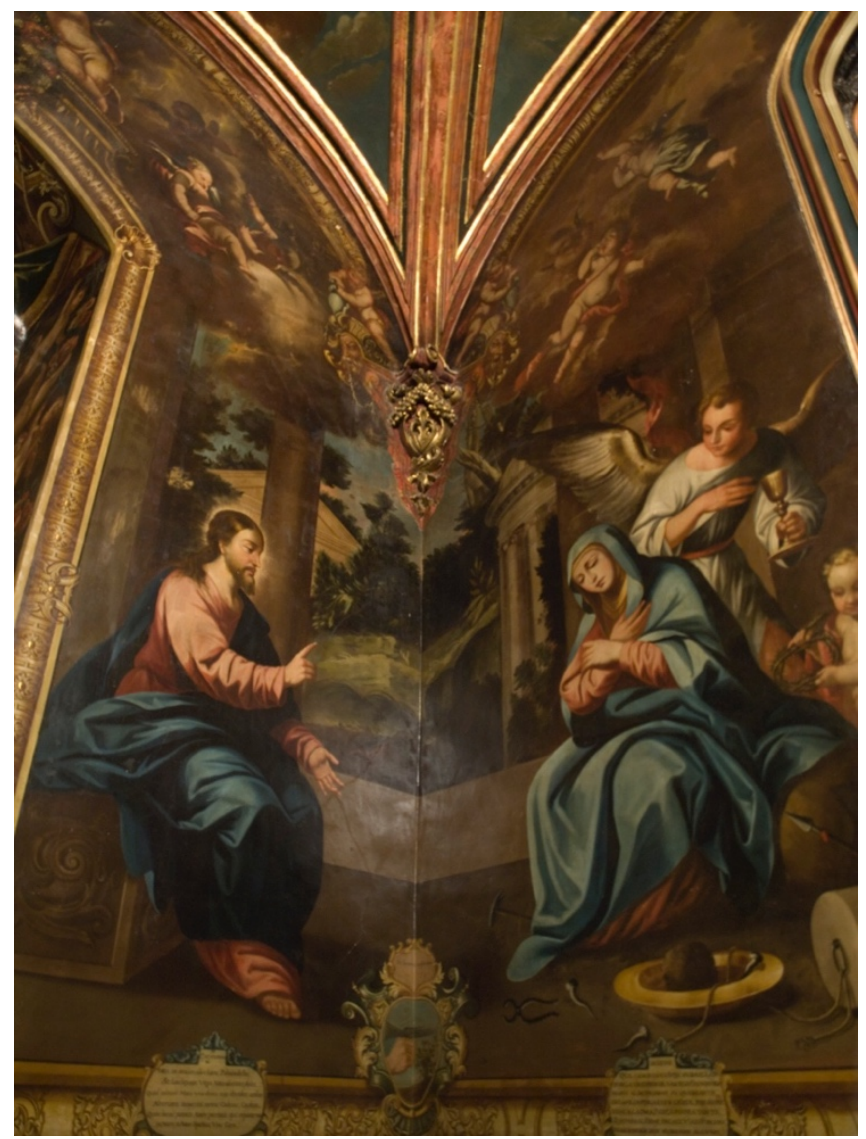

Fig. 4. José Hidalgo y Juan de Medina. Presunción de la Pasión. Basílica de Nuestra Señora de las Angustias, Granada.

/El amor y el dolor, pero sin tasa, sale la espada de la Sacra Voca, y a la Madre en el alma ya le toca".

La Circuncisión está protagonizada por el Sacerdote y el Niño ${ }^{30}$ que alza la mirada mientras caen pequeñas gotas de sangre sobre el paño blanco. Detrás de ellos José permanece con una vela junto a María y, a sus pies, un ángel llora amargamente como preludio a la Pasión y muerte de Jesús junto al siguiente mensaje de la octava:

"Apenas del Oriente se lebanta/El sol dexando el Gremio del Autora/quando vierte de luces copia tanta/que con mucho arrebol el cielo dora/Al sol Jesus aun niño se adelanta/En Sangre esmalta el nombre que se adora/María Dio de Amor estas estrenas/Yapenas nace quando nace apenas".

Las siguientes composiciones flanquean la ventana de la sala. En primer lugar se percibe el Sueño de José, concretamente cuando el Árcángel le avisa en sueños con las siguientes palabras: "Surge et accipe puerum, et matre ejus, et fuge in Egyptum ${ }^{31}$. Math 2 V13." A través de esta escena, se alude al episodio de la Huida a Egipto aprovechando la totalidad del espacio al focalizar su atención en los dos personajes principales y apoyándose en el discurso de la octava:

"Con las alas por Remos Surca el Viento/El ave Cauta, temiendo la Saeta,/Y Joseph del temor sacando aliento,/A Egipto pone de su afan por meta;/Mal Herodes te sale el vano intento,/Un Ángel esta fuga la decreta;/Mas si tu flecha al Niño no le hiere/A su Madre lastima, por que quiere".

${ }^{30}$ En la filacteria se lee "Vocatum est nomen ejus Jesus"-“Fue llamado Jesús”. Lc 2,13,21.

31 "Levántate y toma al niño y a su madre, y huye a Egipto". 


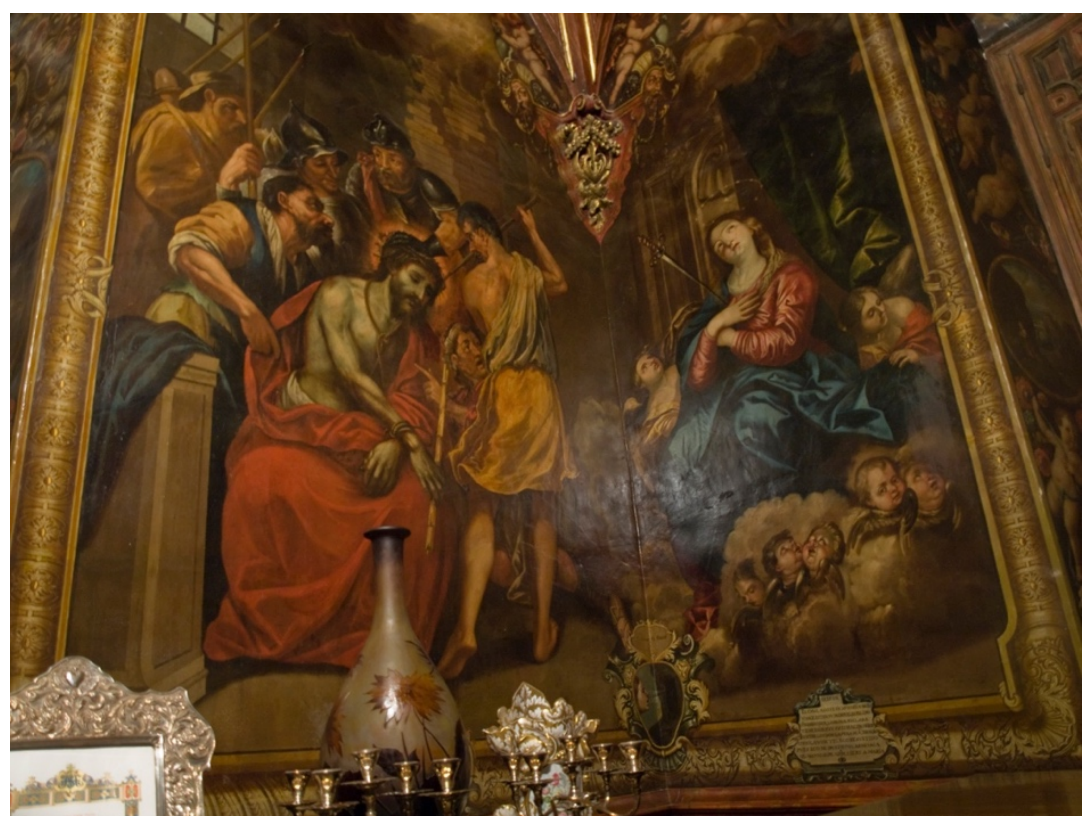

Fig. 5. José Hidalgo y Juan de Medina. Dolorosa y Eccehomo. Basílica de Nuestra Señora de las Angustias,

La representación de Jesús entre los Doctores se encuentra en el otro lado de la ventana. En ella se detalla el momento en el José y María encuentran a Jesús sobre un púlpito y le dicen: "Fili. Pater tuus, et Ego dolentes querebamus te ${ }^{32}$. Luc V.48", mientras los doctores lo miran estupefactos. Una situación de angustia que es descrita por la octava:

"Tocada del Yman la Aguja gira/En busca de su Norte presurosa, Nada la aquieta: a su centro aspira; /Así la dulce María pesarosa/ Perdido busca al Hijo, en quien respira;/Su amor, hasta encontrarlo, no reposa:/Que dolor, Cielos Santos, que tormento/Vivir, muriendo, sin tener aliento".

La última escena versa sobre la actuación de Jesús en el templo y se encuentra junto al arco de acceso al camarín. Aquí aparece Jesús, ya adulto, diciendo ante su madre, y varios seguidores "Possum destruere Templum Dei, et post triduum reedificare illud"Mat 26 V6. ${ }^{33}$ Uno de los aspectos más significativos de esta pintura es que el mensaje de la filacteria sostenida por el ángel está escrito completamente al revés: Lo que batur templo corpore sui,

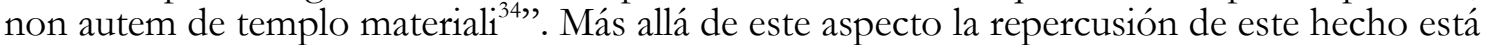
relacionado intrínsecamente con la construcción del templo por lo que en la octava se escribe lo siguiente:

"Fabrica noble, templo sacrosanto/Dios en la tierra para si fabrica, sobre el Cielo la Mole con espanto/Morando Dios allí lo santifica, la ruina de este Templo gran quebrando/a la madre data que lo edifica/pues siendo María perus Templo/al desplomarse su dolor contemplo."

Con esta escena se concluye el primer ciclo, ascendiendo por las escaleras hasta el camarín de Virgen.

32 "Hijo. Tu padre y yo te hemos buscado con angustia".

33 "Puedo destruir el templo de Dios, y reconstruirlo después de tres días". Curiosamente este mensaje está completamente escrito al revés.

34 "Él hablaba del templo de su cuerpo, pero no fuera del templo que es material". 
Antecamarín del lado del Evangelio: Pasión y muerte de Cristo (1741-1742) ${ }^{35}$.

En esta estancia se confirman los preludios manifestados en el ciclo anterior a través de la representación de los misterios.

La lectura del programa continúa en el lado izquierdo del arco principal y se concluye en la salida con esbozos alusivos a la Resurrección.

La primera gran composición es Presunción de la Pasión, una escena doble en la que la esquina de la habitación marca el punto de encuentro. En ella Jesús se despide de su madre sentado sobre un bloque bellamente esculpido, mientras ella permanece con los brazos en cruz en señal de recogimiento. Así lo describe la Octava:

"Qua generosa u(ste)d que suda el l(1)an(to)/por la yncisión del vastago frondoso/Maria al despedirse, su quebranto/engasta en perlas, y en cristal presioso:/nunca la dura fuerça podra tanto,/q(ue)rompa el firme enlace, y ia es forçoso,/ayan de morir dos muriendo alguno/pues uno(.) amor los dos, en solo uno".

la siguiente composición pretende crear un efecto doble a través de la conjunción de la imagen de la dolorosa y el eccehomo, pero no alcanza el efecto deseado a pesar de las letras de la octava:

"El cruel azote en xto(cristo)resonaba/y hace eco en su madre el estal(1)ido/JHS(Jesus) erido esta reina esclaba/tiene en jesus su corazón her(i)d(o) /entre los dos la pena se a(l)ternaba/como la vox y el eco resturtido/pues eco de durisissma armonía/cuando hiere a ihs hiere a Maria".

Así pues María, Dolorosa, aparece levantando la mirada mientras un puñal está clavado en su pecho. Por su parte la composición del Eccehomo ${ }^{36}$ emplea una forzada perspectiva que provoca que el tamaño de Jesús sea desproporcionado en relación con los personajes que lo humillan. No obstante, el objetivo real sería suscitar el arrepentimiento, la compasión y el dolor ante una imagen de Jesús coronado, sujetando la caña y vestido con el manto púrpura.

Junto a esta escena se encuentra una hornacina que acoge un bello escudo con la leyenda "Real Hermandad de Nuestra Señora de las Angustias" que aparenta estar sobre un jarrón de flores posado sobre un suelo de losas blancas y negras y flanqueado por dos ángeles arrodillados que lloran amargamente mientas sostienen dos velas.

Finalmente, este lienzo arquitectónico se completa con la composición de El camino al Calvario, donde Jesús lleva la cruz a cuestas. Esta pintura nos remite al siguiente texto del evangelio de Lucas: "Mientras lo conducían, echaron mano a un tal Simón de Cirene, que volvía del campo, y le cargaron la cruz para que la llevase detrás de Jesús" (Lc 23,26). La belleza de esta escena se descubre en las miradas de la Madre y su Hijo, mientras que la carga sentimental recae sobre María Magdalena, que seca sus lágrimas junto a Juan y se lee en la Octava:

"La flor que los calores estivales/acostaron por fin en la ribera, se/dobla ante los líquidos cristales/del lago que lozano la volviera: así María, muriendo por los males/que el gentílico pueblo le impusiera/do mitigarlos con afán procura y/haya un nuevo dolor en la amargura".

35 Al comparar minuciosamente los trabajos murales de Medina, de los que actualmente tenemos constancia, se evidencian las analogías con en las pinturas al fresco realizadas por Medina en el crucero de la iglesia del monasterio de San Jerónimo de Granada en 1723. En estas composiciones el también recurrió a los grabados de Rubens para la representación de las escenas de la Crucifixión, por tanto es posible diferenciar la paleta de Medina en la mayor parte de las escenas del Ciclo de la Pasión y muerte de Cristo.

${ }^{36}$ Cristo está siendo azotado por los soldados antes de crucificarle. Los verdugos suelen ser deliberadamente feos, y disfrutan del momento, mientras que Jesús se identifica con lo bello y luminoso. Ecce Homo "este hombre". Después de ser azotado Poncio Pilatos lo saca al balcón del Pretorio y lo muestra diciendo "Aquí tenéis al hombre (Jn 19,5), ofrecido para indultarle la gente clama para que sea crucificado. Finalmente se lavó las manos y lo entregó para cumplir los deseos del Sanedrín y los judíos. 
La Lanzada es la composición de mayor tamaño en este ciclo. De hecho la Crucifixión es el momento "más importante de la Pasión, pues con su sacrificio en la cruz culmina su misión redimiendo a la humanidad del pecado original ${ }^{37 ",}$.

Para realizar exitosamente esta representación, los pintores siguieron rigurosamente los modelos de Rubens ${ }^{38}$, añadiendo personajes secundarios. Por ello encontramos bajo un cielo de tinieblas a un longinos avanza clavándole a Jesús una lanza en el costado. A los pies de la cruz y en el lado derecho, los seguidores de Cristo se apenan por la muerte del Mesías, sobre todo sus más allegados: María Magdalena que, arrodillada y abrazando la cruz, eleva sus brazos y su mirada hacia Jesús, Juan que seca sus lágrimas con un paño y María, que aparece triste e impactada ante un momento de tal intensidad, incapaz de expresar los sentimientos; porque en cierto modo, la muerte de Cristo, es el final de la Pasión de María, el mayor sacrificio que le había sido encomendado. Así lo resume la octava:

"Musico es el amor y dulcemente/ por cuerdas pulsa fieles coraçones:/cithara la cruz es, Christo pendiente/ le da cuerdas en rigidas tenciones/ es cithara María, que pendiente consuena sin diversas pulsaciones, / cithara herida es christo, y como unida / a M(aria) suena por la misma herida".

En el lateral del arco del camarín, se encuentra la última escena de grandes dimensiones del ciclo de la Pasión: el Descendimiento de Cristo de la cruz. en el monte del Gólgota. En esta escena las líneas de composición son definidas por la Cruz, la escalera y el cuerpo muerto de Cristo, cubierto por un paño, trasladado por José de Arimatea, Nicodemo y Juan. Por su parte, María Salomé y María Magdalena lloran mientras María permanece arrodillada sujetando el brazo de su hijo. Una escena que es descrita así por la última octava:

"Muere el sol y la luna berdadera/afecta posseción de resplandores/muere Jesus, y la luna de otra esfera/su madre hereda luces entre horores/ suscesión de la luna

\footnotetext{
37 Carmona Muela, 2008: 149.

38 Para realizar los programas estipulados había una serie de modelos que los pintores utilizaban, y en este caso podemos comprobar que los pintores del Camarín tuvieron acceso a los bocetos de Rubens, que ya tenía Juan de Sevilla.
} 


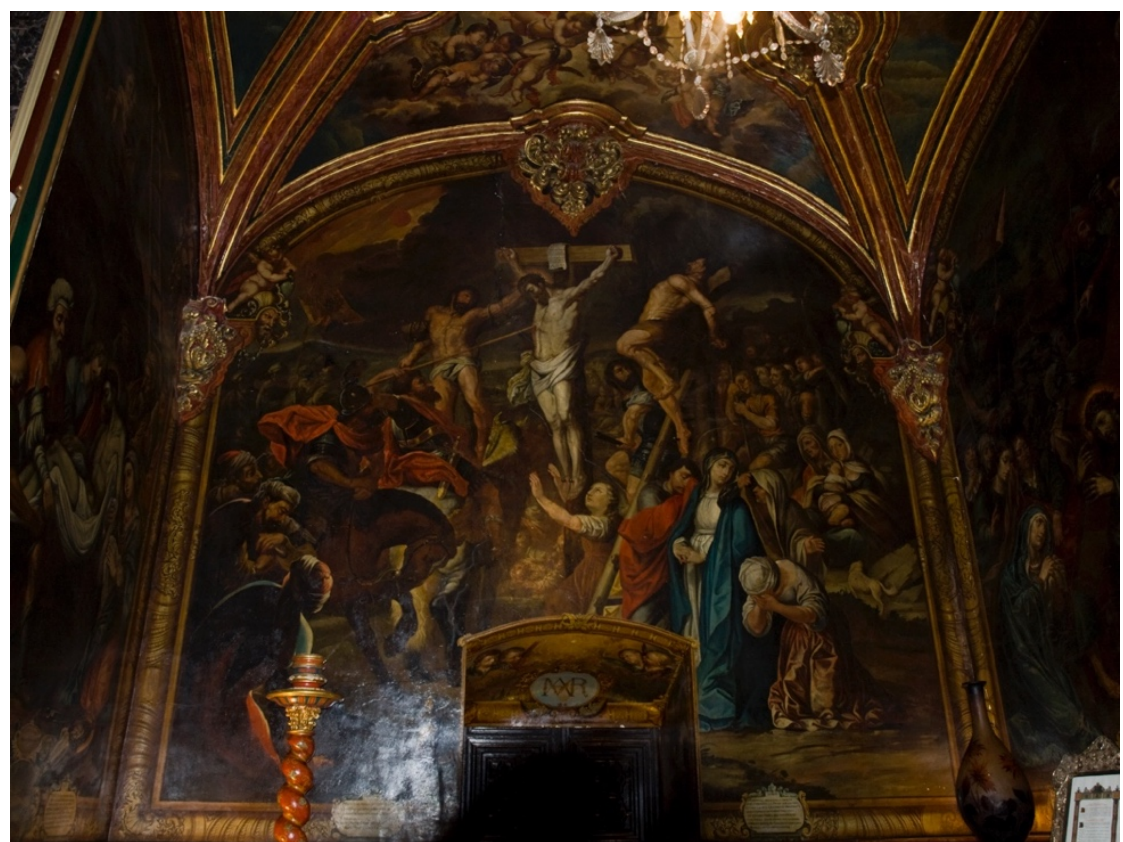

Fig. 6. José Hidalgo y Juan de Medina. La lanzada. Basílica de Nuestra Señora de las Angustias, Granada.

verdadera/el cuerpo solar es, y sus fulgores/ y por derecho que del arbol pende/va el de hijo a madre pues de allí desciende”.

Como apéndice del discurso iconográfico, en la puerta de salida se encuentra una luminosa estrella donde se lee "Lumen in Coelum (may) "3\%" rodeada por varias cabecitas aladas de querubines, ahora sonrientes. Las escenas del Sepulcro vacio se esbozan en las dovelas del arco a través de una serie de pinceladas sueltas que perfilan los trascendentales momentos referentes a la Resurrección.

Finalmente merece una mención especial la belleza de las bóvedas de crucería que cubren los antecamarines salpicadas por angelitos portadores de los atributos de la Pasión, y que envuelven estas magníficas composiciones invitando al recogiendo y a la reflexión a través de la armónica conjunción entre palabra e imagen.

\section{Valoración del conjunto.}

La decoración pictórica de los antecamarines de la Basílica de Nuestra Señora de las Angustias evoca a la perfección la simbiosis entre los cánones estéticos del Barroco y la espiritualidad granadina de la primera mitad del siglo XVIII.

Para realizar una obra de tales dimensiones resultó imprescindible la contribución de Juan de Lizana. En efecto, gracias a su apoyo financiero se sufragó la participación de los artistas más prolíficos de la ciudad: culminar la fábrica arquitectónica y recrear pictóricamente un minucioso discurso iconográfico sustentado en los pilares fundacionales de la Hermandad.

En definitiva, nos encontramos ante un complejo discurso simbólico que conduce a los devotos por un sendero de recogimiento espiritual, a través de la creación de una obra de arte total que acrisola la visión de las pinturas y la lectura de los textos, con el sonido de la música de los órganos y el olor incienso. Una atmósfera de trascendental devoción religiosa.

39 "Luz en el cielo". 


\section{Bibliografía}

Antequera, Marino (1973): La Virgen de las Angustias. Granada: Obra Cultural de la Caja de Ahorros de Granada.

Carmona Muela, Juan (2008): Iconografía cristiana. Guía básica para estudiantes. Madrid: Akal.

Chavarino García, María Jesús (1986): Noticias histórico-artísticas en el Archivo de la Hermandad de Nuestra Señora de las Angustias. Memoria de licenciatura bajo la dirección de Antonio Moreno Garrido. Granada.

Gallego Burín, Antonio (1982): Granada. Guía artística e histórica de la ciudad. Granada: Quijote.

Giménez Serrano, José (1846): Manual del artista y el viagero en Granada, Granada: J.A. Linares.

Gómez Moreno, Manuel (1982): Guía de Granada. Granada: Universidad de Granada.

Gutiérrez Galdo, José (1983): La Virgen de las Angustias, Patrona de Granada. Granada: Santa Rita.

Isla Mingorance, Encarnación(1989): La Virgen de las Angustias. Granada. E.Isla.

Jiménez Alarcón, Margarita: "Real Academia de Bellas Artes de Nuestra Señora de las Angustias de Granada. Nota para su historia”. En: Boletín Real Academia de Bellas Artes de Granada, 1, Granada.pp.13-25.

López Guadalupe, Miguel Luis, López Guadalupe, Juan José (1996): Nuestra Señora de las Angustias y su Hermandad en la Epoca Moderna. Notas de historia y arte. Granada: Comares S.L.

Sánchez Sarabia, Diego (1777): Compendio histórico del origen y culto en Granada de Nuestra Señora de las Angustias. Granada: Imprenta de la Santísima Trinidad. 